\title{
¿Quiénes son las parteras munduruku? Pluralismo médico y autoatención en el parto domiciliario entre indígenas en Amazonas, Brasil
}

\author{
RAQUEL PAIVA DIAS-SCOPEL Y DANIEL SCOPEL
}

A partir de la etnografía sobre embarazo y parto entre los indígenas munduruku, se verificó que los saberes considerados tradicionales no se restringen a los especialistas y se comparten entre varias personas de la familia, en especial, entre las mujeres mayores, quienes pueden ser reconocidas como parteras o no. La actuación de las mujeres mayores y parteras munduruku ocurre en el contexto de participación creciente de profesionales de la salud y no se presenta como un simple antagonismo entre las formas de atención tradicional y biomédica, lo cual implica la articulación entre actores sociales con potencial para promover intercambios de saberes. Al mismo tiempo, las prácticas indígenas difieren de manera radical del paradigma biomédico y se perciben como necesarias para la salud del bebé y de la familia.

PALABRAS CLAVE: indígenas sudamericanos, etnografía, parto domiciliario, pluralidad médica, parteras

Who Are the Munduruku Midwives? Medical Pluralism and Self-Care in Home Delivery among the Indigenous People of Amazonas, Brazil

Raquel PaIVA Dias-SCOPEL Fundação Oswaldo Cruz, Campo Grande, Mato Grosso do Sul, Brasil raquel.scopel@gmail.com

DANIEL SCOPEL

Fundação Oswaldo Cruz, Instituto Leônidas \& Maria Deane, Manaos, Amazonas, Brasil daniel.amazonia@gmail.com

Traducción: Mauricio Pardo Rojas
Based on the ethnography on pregnancy and delivery among the Munduruku, we observed that knowledge regarded as traditional is not in fact restricted to specialists but rather shared among many people in the family, particularly elderly women, who may or may not be viewed as midwives. The work provided by Munduruku elderly women and midwives takes place in a setting of growing participation of health professionals, which does not take the form of a simple antagonism between the traditional and biomedical modes of care, implying interaction among social actors who have the potential to promote knowledge exchange. At the same time, indigenous practices are radically different from the biomedical paradigm and are perceived as mandatory to ensure the health of both the baby and the family.*

KEYWORDS: South American indians, ethnography, home delivery, medical pluralism, midwives 


\section{Introducción}

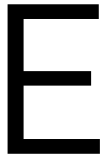

n Brasil, la Política nacional de atención a la salud de los pueblos indígenas (PNASPI; Fundação Nacional de Saúde, 2002) establece la atención diferenciada como principio de atención primaria prestada en las aldeas. La PNASPI propone la oferta de servicios adecuados a las especificidades geográficas, epidemiológicas y socioculturales de los pueblos indígenas y recomienda la articulación con sistemas tradicionales de salud. Aunque este instrumento reconoce la diversidad y las especificidades de las prácticas indígenas, los estudios etnográficos muestran que las políticas públicas todavía reproducen una imagen caricaturesca y estereotipada de los sistemas de salud tradicionales (Langdon, 2007; Ferreira, 2013; Scopel, 2013; Dias-Scopel, 2015).

A partir del año 2000, la categoría de partera tradicional reapareció como objeto de políticas públicas en Brasil, con el programa Trabajando con Parteras Tradicionales, que objetiva la valorización de los sistemas tradicionales de salud, la formación y capacitación de agentes locales y su inclusión en el servicio de atención primaria para reducir la mortalidad materna y neonatal (Ministério da Saúde, 2010).

En el estado de Amazonas, la implementación del programa se llevó a cabo desde 2008 y se buscó registrar y capacitar parteras tradicionales (Silva, 2017). La iniciativa tiene los méritos de hacer manifiesta la intervención de otros actores sociales en la atención de la salud de la mujer, además de los servicios prestados por profesionales de la salud de tipo biomédico; exaltar su respeto y valoración, y proponer una articulación con las parteras tradicionales como una forma de ampliar las medidas de atención primaria en salud orientadas a obtener mejores indicadores. Sin embargo, hay puntos que merecen ser repensados, en especial cuando estas acciones se extienden a las poblaciones indígenas.

\footnotetext{
* Traducción al inglés de Amanda Francisco.
} 
Con base en el problema teórico del contexto de pluralidad médica en Latinoamérica, en particular en el campo de la salud indígena brasileña, varios autores se cuestionan una serie de conceptos y preconceptos relacionados con la polarización entre las categorías "moderno" y "tradicional" (Menéndez, 1987; 2009; Langdon, 2007; Garnelo, 2003). El concepto de tradicional, cuando se define como oposición a lo moderno, no se sostiene etnográficamente, pues en la práctica, los actores sociales acumulan una diversidad de saberes. Entre los munduruku, los saberes tradicionales no son estáticos ni herméticos, sino que se encuentran en reformulación continua (Scopel, Dias-Scopel y Langdon, 2018).

En este artículo describimos las prácticas de atención y autoatención durante el embarazo y parto entre los munduruku, con el objetivo de comprender quién es la partera munduruku. El enfoque etnográfico es relevante para reflexionar sobre las limitaciones y equívocos derivados de una tipificación que opone las categorías tradicional/moderno, característica en las prácticas de los profesionales de la salud (Langdon, 2007) y en las políticas públicas para los pueblos indígenas (Ferreira, 2013). Argumentamos que, desde un punto de vista indígena, el contexto de pluralidad de formas de atención entre los munduruku se distingue por la posibilidad de articulación entre las formas tradicionales y biomédicas, al menos en lo que respecta a la atención de la gestación y el parto.

Con la ampliación de la cobertura de los servicios de salud biomédicos, el parto hospitalario en entidades públicas se ha convertido en un recurso accesible para las mujeres indígenas brasileñas. Aunque todavía no existe un análisis nacional del contexto del parto entre indígenas, a partir de relatos en investigaciones etnográficas podemos señalar que hay casos extremos en los que la totalidad de los partos es hospitalaria (Gigghi, 2015) o domiciliaria, y en otros hay variaciones de lugar (Wakanã y Pérez, 2007).
En esta ocasión, analizamos sólo el contexto del parto domiciliario munduruku, pues en otro trabajo examinamos la inserción de las gestantes en la atención básica y concluimos que la tendencia a la hospitalización del parto es una realidad no consolidada en este grupo, a pesar de que algunas indígenas demandan la realización de cesáreas y esterilización femenina (Dias-Scopel, Scopel y Langdon, 2017). Entre 2008 y 2010, 77 de los 143 partos ocurridos fueron domiciliarios - 54\%-, es decir, los partos fuera del ambiente hospitalario son frecuentes. El caso munduruku muestra que la medicalización de la gestación y el parto no es un proceso acabado. A pesar de las actitudes ambiguas de los profesionales de la salud, los indígenas buscan integrar sus prácticas tradicionales a la creciente y más constante presencia de servicios biomédicos en las aldeas, al menos desde la implantación de la PNASPI. Entre los munduruku, el parto domiciliario es conducido por una mujer indígena experimentada o por una partera que auxilia a la gestante. Sin embargo, el parto no es un evento aislado, sino que forma parte de un proceso más amplio, cuyo éxito, como veremos, depende de las prácticas de autoatención llevadas a cabo desde el comienzo de la gestación bajo la guía de mujeres indígenas que pueden ser reconocidas o no como parteras tradicionales.

\section{Trabajo de campo y metodología}

El pueblo munduruku está formado por aproximadamente 10000 personas que viven en Tierra Indígena de los estados de Pará, Mato Grosso y Amazonas. En Amazonas, cerca de 2500 munduruku ocupan la Tierra Indígena Kwatá-Latanjal (TIKL), en 33 aldeas distribuidas a lo largo de los dos ríos que la atraviesan, 12 aldeas en el río Mari-Mari y 21 en el río Canumã. Las selvas de la TIKL están bien conservadas y tienen abundancia de animales y peces. Todos hablan portugués y sólo algunos viejos 


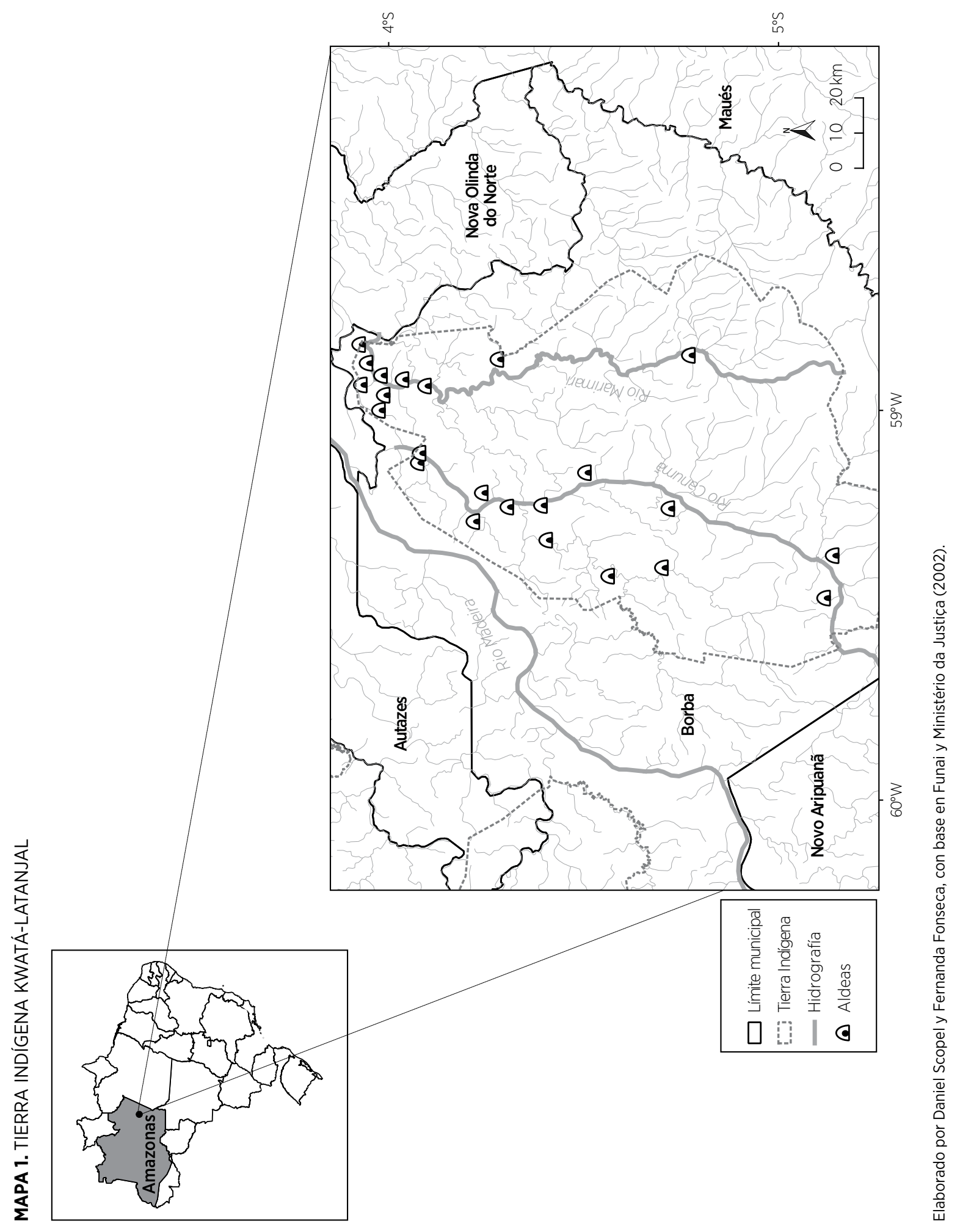


hablan munduruku, del tronco lingüístico tupi. La ciudad más cercana a la TIKL, donde se ubica el hospital, es Nova Olinda do Norte. Los indígenas van a la ciudad con frecuencia para comprar víveres en el comercio local, vender productos agrícolas y extraídos del bosque y recibir beneficios sociales.

Nuestro trabajo de campo etnográfico se llevó a cabo entre 2009 y 2011, así como en 2017, para sumar casi diez meses que incluyen tres estadías largas en la TIKL. Las técnicas de obtención de los datos principales fueron la observación participante, el levantamiento de genealogías, el registro de narrativas y entrevistas. Utilizamos seudónimos para preservar la identidad de los interlocutores.

A partir del levantamiento genealógico, la morfología de las aldeas munduruku puede describirse por la ocupación territorial de las familias elementales, que se organizan en grupos domésticos comensales y en familias extensas, compuestas por un conjunto de familias elementales que comparten el mismo segmento residencial, es decir, construyen habitaciones contiguas. Así, es posible observar una correlación entre la ocupación territorial y la organización social basada en redes de parentesco. La familia elemental es la unidad mínima de organización del trabajo en la producción y consumo de harina de yuca, su principal producto agrícola. Cada familia elemental tiene su casa y cultiva una chagra propia, en la que se planta yuca. Las familias elementales se organizan en un sistema de trabajo comunal que refuerza los lazos entre ellas. Los munduruku también se dedican a la caza y la pesca artesanal para subsistir. Pocas personas reciben salarios, como profesores o agentes de salud.

Empezamos la etnografía en un segmento residencial en el que compartimos vivienda con una familia nuclear. Después la extendimos hacia otros segmentos en las aldeas de Niterói, Frontera y Cajual. La observación participante incluyó desde actividades diarias hasta momentos festivos o ceremoniales, como trabajos del ámbito de la casa
— cocinar, buscar agua, limpiar el terreno, etc.prácticas de autoatención en salud y enfermedades, trabajo en la chagra, producción de harina, grupos de conversación, consultas a los curadores payés, velorios, festividades, reuniones de salud y asambleas políticas.

La observación participante se realizó con nueve mujeres de la vecindad que quedaron embarazadas y cumplieron la dieta del posparto. Recolectamos narrativas de 24 mujeres sobre sus experiencias de embarazo, parto domiciliario y hospitalario. Hicimos entrevistas con profesionales de la salud y hombres munduruku — padres, profesores, agentes indígenas de salud (AIS), consejeros de salud, curadores payés, caciques jóvenes y viejos-.

De manera análoga, se observaron las actividades del servicio de salud oficial, a cargo de un equipo multidisciplinario de salud indígena (EMSI), con sede en Polo Base Kwatá o aldea Kwatá. El polo base funciona como un centro de salud y es la primera referencia para el EMSI que actúa en las aldeas del río Canumã. El EMSI está compuesto por una enfermera coordinadora, un médico, un dentista y técnicos en enfermería y AIS. Las demás aldeas sólo cuentan con AIS (Scopel, Dias-Scopel y Langdon, 2015). Las indígenas van a la ciudad de Nueva Olinda del Norte para acceder al parto hospitalario. El trayecto de la aldea a la ciudad es por río.

De la experiencia de Raquel, coautora de este texto, que estaba embarazada, surgió información importante. Al convivir con ella, hombres y mujeres munduruku compartieron vivencias, dieron consejos e hicieron interpretaciones sobre los sucesos alrededor del embarazo. Gracias al trabajo etnográfico fue posible percibir que en la sociedad munduruku los saberes sobre embarazo, parto y posparto están ampliamente difundidos entre hombres y mujeres, y las prácticas de atención relativas a la gestación y el parto no están restringidas a los especialistas y las parteras (Scopel, Dias-Scopel y Wiik, 2012). 


\section{Prácticas de atención a la salud de la mujer munduruku}

\section{EN EL EMBARAZO}

Las mujeres munduruku pueden contactar una pluralidad de actores sociales durante el embarazo y el parto. Algunos fundamentan su legitimidad en las relaciones de parentesco, es decir, actúan en el interior de la familia extensa, la cual reconoce y legitima su experiencia. Éste es el caso de ciertas mujeres de la familia extensa, cuyo prestigio se sustenta en la experiencia de atención a las otras mujeres de su familia. Los llamados curadores payés, hombres y mujeres, son reputados por dominar conocimiento sistematizado sobre el mundo invisible y espiritual. $\mathrm{Su}$ legitimidad se construye y mantiene con el ejercicio de prácticas chamánicas. Además, están aquellos cuya legitimidad reside en el dominio de saberes y prácticas biomédicas, como los profesionales de la salud que trabajan en la aldea.

"Agarrar la barriga" es una práctica central de atención a la salud de la mujer en general y de las embarazadas en particular. Siempre que las mujeres munduruku adultas sienten un malestar o sospechan que ha comenzado el embarazo, se someten a la práctica de agarrar la barriga por otra mujer mayor de la familia extensa, una partera o un curador payé. Agarrar la barriga consiste en masajear y palpar el vientre con aceites vegetales extraídos de la selva o con cremas y aceites comprados en la ciudad en farmacias o supermercados.

En la familia extensa con la que convivimos, Araci era una de las mujeres más viejas y sabía agarrar la barriga. Naná, que vivía en el mismo segmento residencial, nos contó que acudió a Araci para que le agarrara la barriga para "halar la madre del cuerpo". Para los munduruku, las mujeres poseen una madre del cuerpo que se aloja en el vientre, debajo del ombligo. La madre del cuerpo no es el útero. Aunque tiene materialidad para ser palpada durante los masajes, se describe como una especie de fuerza responsable de la salud y vida de las mujeres. El desplazamiento de la madre del cuerpo fuera de su lugar puede causar enfermedades y muerte. Cuando se siente malestar, dolor de cabeza, fiebre, mareo, dolor abdominal, cólico, diarrea o dolor en el cuerpo, lo primero que hacen las mujeres munduruku es buscar a alguien que les agarre la barriga para comprobar si la madre del cuerpo está en su lugar. De manera complementaria, las mujeres también pueden hacer uso de infusiones y "remedios de la selva".

Durante el embarazo, la madre del cuerpo se desplaza para dar lugar al bebé. Por eso, las mujeres van con alguien que les agarre la barriga. Las primeras en confirmar el embarazo son las que saben agarrar la barriga. Al sospechar el embarazo, las mujeres a quienes acompañamos fueron con una de las más viejas de la familia extensa para que les agarrara la barriga.

Después de confirmar el embarazo, las que vivían en la aldea Kwatá, sede del EMSI, buscaron los servicios biomédicos para empezar el control prenatal, una de las acciones previstas por el Ministerio de Salud (MS). En el trabajo al que ya hemos hecho referencia analizamos la participación de las mujeres munduruku en el programa biomédico prenatal y las negociaciones sobre la elección del lugar del parto (Dias-Scopel, Scopel y Langdon, 2017). Este programa es ejecutado por técnicos de enfermería - hombres y mujeres-, la enfermera y el médico. En 2011, sólo 75\% de las 39 mujeres embarazadas en las 21 aldeas del río Canumã recibieron seguimiento biomédico del equipo, con al menos una consulta. El Ms brasileño recomienda un mínimo de seis. Para las mujeres indígenas que viven en las aldeas del río Canumã, la centralización de los servicios biomédicos y el EMSI en la aldea Kwatá sobresale como una de las dificultades para ingresar y permanencer en el programa. De acuerdo con DiasScopel, Scopel y Langdon (2017), las motivaciones para elegir el lugar del parto tomaban en cuenta 
que las aldeas munduruku del Canumã están a una distancia considerable del hospital más cercano, y el viaje, que se hace sólo por río, puede durar de dos a ocho horas. Se observó que la elección del lugar de parto — domicilio u hospital - se construye desde la gestación, por lo tanto, involucra negociaciones entre varios actores sociales: gestante, cónyuge, mujer mayor de la familia extensa, parteras, payés y profesionales de la salud. Aunque estos últimos incentivaban el parto hospitalario, las negociaciones se sustentaban en una gama de expectativas diversas; no eran simples imposiciones biomédicas, incluían criterios compartidos por profesionales de la salud e indígenas, como estimaciones de edad, estado de salud y experiencias anteriores de la gestante, posición del bebé y consulta a payés o parteras.

Queremos subrayar que los profesionales de la salud apoyan el parto hospitalario en especial cuando atienden a mujeres primerizas: "a todas las primíparas las enviamos al hospital”, afirmó Alice, una de las técnicas de enfermería. También criticó el trabajo de las parteras: "mandan pujar antes de tiempo, mientras que en el EMS se evalúa por la dilatación". Se refería a una experiencia de parto domiciliario en la que participó: "cuando llegué allí, la mujer ya estaba tirada, cansada" (entrevista con Alice, aldea Laranjal, 16 de febrero de 2010). El discurso de Alice sobre el trabajo de la partera no es unánime en el EMSI. La enfermera y el médico (entrevista, aldea Kwatá, 31 de enero de 2010) adujeron la necesidad de "trabajo en asociación" con las parteras, curadores payés y AIS.

A pesar de los discursos ambiguos de los profesionales de la salud, que incentivan la medicalización del parto en ciertos momentos, las mujeres que ingresan a los servicios de salud biomédica para realizar el control prenatal continúan con las prácticas de agarrar la barriga y halar la madre del cuerpo, entre otras, como restricción alimentaria, prescripciones morales y baños (Dias-Scopel, Scopel y Langdon, 2017).
Agarrar la barriga es una práctica recurrente durante todo el embarazo y el posparto para mantener la salud y obtener información sobre la madre y el bebé. En ciertos momentos, se hace más de una vez al mes. Para los munduruku, las mujeres pueden quedar embarazadas de animales-espíritus subacuáticos —delfines y "encantados"- y dar a luz bichos o animales (Dias-Scopel, 2015). Las mujeres que saben agarrar la barriga identifican si es embarazo de gente o de animal por la consistencia de lo que palpan. Dicen que el niño, la gente, el feto humano, tiene consistencia firme, semejante a un objeto sólido y entero. El hijo de animal, por el contrario, tiene una consistencia flácida e inestable y se deslíe al ser palpado, es decir, al toque de las manos el contenido se "esparce entre los dedos", "se esparce en la barriga", "como si se deshiciera" (notas de campo, 20 de marzo de 2011). Este saber reside en la investigación de la anatomía del feto a partir de un modelo cultural del cuerpo humano que las mujeres mayores de la familia extensa aprendieron con otras mujeres, sobre todo con la práctica de agarrar la barriga de sus hermanas, hijas, sobrinas, nietas, entre otras parientes. Además de la anatomía del feto, hay otros signos y síntomas indicativos de la gestación de un hijo de animal, como adelgazamiento, debilidad, dolores abdominales y en la cadera.

El análisis de los movimientos fetales también es un saber que informa sobre la salud en el embarazo. Las mujeres munduruku saben que el hijo de animal no se mueve. Iara, por ejemplo, fue a que su cuñada le agarrara la barriga porque el bebé no se movía al final del tercer mes de gestación (notas de campo, 20 de marzo de 2011). Además de señalar un embarazo sano, los movimientos fetales se evalúan para indicar el sexo del niño. Las mujeres que saben agarrar la barriga combinan los conocimientos sobre movimiento fetal con los de la anatomía del feto para predecir el sexo del bebé.

Mujeres y hombres munduruku conversaron con frecuencia con nosotros sobre el embarazo de 


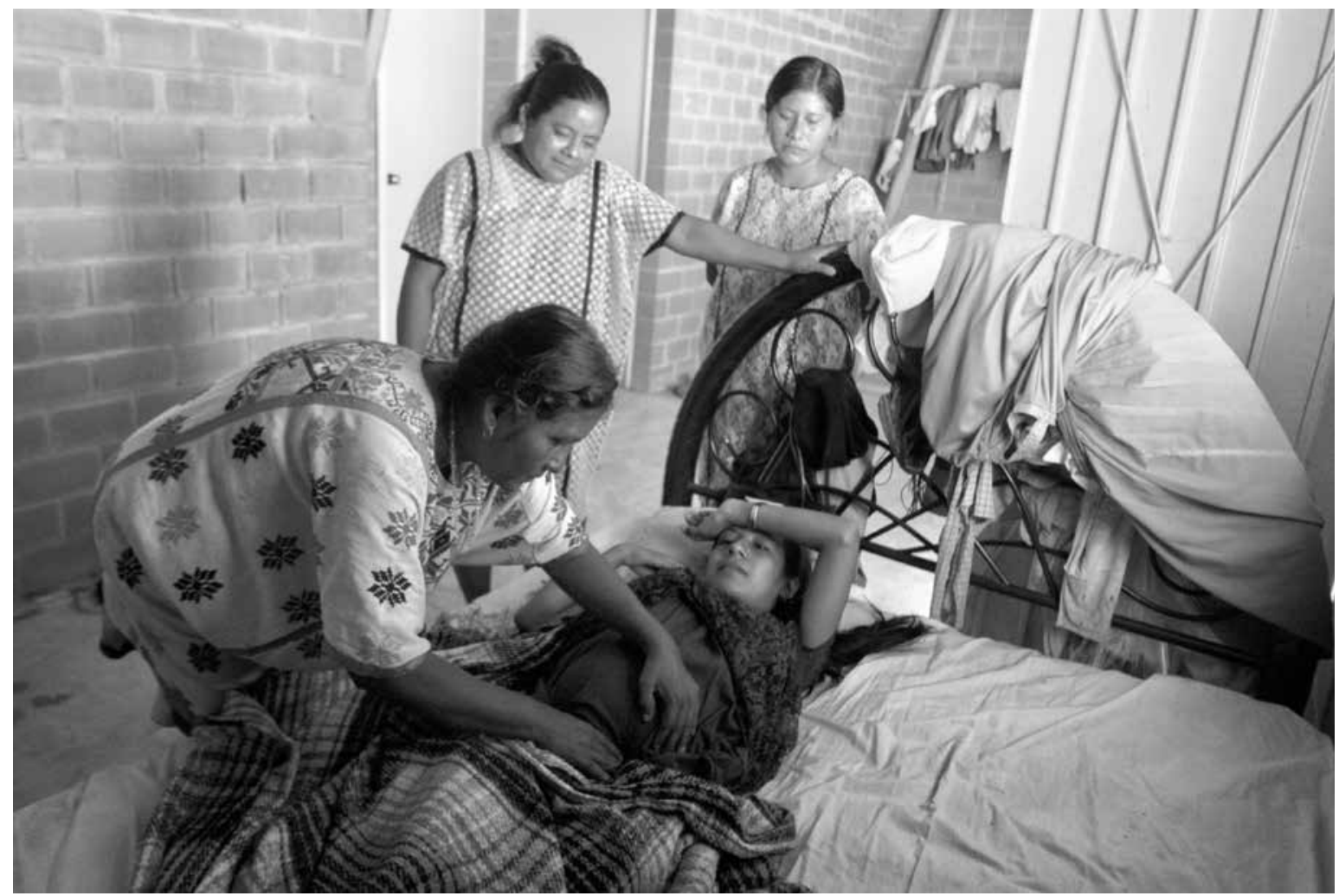

Consuelo Morales PagAZA Ancelma, partera de la comunidad Los Liros, revisa a una mujer que está próxima a parir y enseña a las nuevas generaciones el trabajo de la partería, Costa Chica, Guerrero, 2015.

Raquel, en particular porque alrededor del tercer mes la investigadora no sentía que el bebé se moviera y no se le antojaban ciertas comidas. Entre sus interpretaciones, dijeron que había diferencias entre los fetos masculinos y femeninos relativas a la forma y el movimiento en la barriga de la madre. El feto femenino se forma hasta el quinto mes y no se mueve hasta entonces, tiene consistencia dura, sin forma humana, como una bola, sin tronco, brazos, dedos, pies, etc. El feto masculino, por el contrario, a pesar de ser pequeño, desde el primer mes presenta el cuerpo completo y formado, por lo que la gestante siente los movimientos fetales desde el comienzo del embarazo. Aunque este modelo cultural de la anatomía del feto humano es de conocimiento extendido entre los munduruku, sólo las personas que saben agarrar la barriga — las mujeres más viejas de la familia extensa, las parteras y los curadores payés - dominan la técnica de masajear y palpar, y tienen la habilidad para identificar si el feto es humano, su sexo y la posición en la que se encuentra.

Una vez, las mujeres sugirieron que Raquel fuera a que Araci, nuera de nuestra anfitriona, le agarrara la barriga. Al llegar a la casa de Araci, la investigadora fue llevada a la habitación y colocada sobre la cama. Araci le solicitó que se levantara la blusa hasta la altura de los senos y se bajara las bermudas hasta las ingles. Extendió aceite en sus manos y sobre la barriga de la embarazada e hizo varios tipos de movimientos — circulares, del ombligo hacia la espalda, de los laterales hacia el centro-, y presionó poco a poco todo el vientre. 
La técnica de agarrar la barriga también se utiliza para comprobar si el feto está en la posición correcta. Si es necesario, se coloca en el "nacedero", es decir, con la cabeza hacia abajo. Por ejemplo, observamos que Iara se hizo agarrar la barriga varias veces alrededor de los siete meses de gestación, por varios motivos; entre ellos, por la desconfianza sobre un posible embarazo de animal o por creer que el niño no estaba en la posición correcta. Buscó a su cuñada Araci para que le agarrara la barriga y al curador payé Porã, quien recomendó que tuviera el bebé en el hospital, pues el feto estaba atravesado. Iara siguió con las prácticas de autoatención para reposicionar al bebé y facilitar el momento del parto, al mismo tiempo que asistía a las consultas prenatales en el EMSI. Decidió parir en el hospital, por recomendación del curador payé. Este caso ejemplifica una actitud frecuente entre las mujeres y los especialistas munduruku: solicitan o proponen servicios de atención biomédica durante la gestación, sin que ello implique contradicción con las prácticas de autoatención y atención conducidas por las mujeres mayores de la familia extensa, parteras o curadores payés.

A lo largo del embarazo, las mujeres munduruku toman baños terapéuticos con el objetivo de abreviar el tiempo de expulsión del bebé. Araci, por ejemplo, preparó varios baños para Iara y para Raquel, la investigadora, con una mezcla de hojas de árboles y agua entibiada al sol. Las hojas seleccionadas deben ser lisas, porque tienen la propiedad de facilitar la salida del bebé. A diferencia del baño para higiene personal en el río o cerca del pozo, el baño de hojas debía tomarse dentro de la casa y una parte de la infusión puede beberse. En el caso específico de la preparación de los baños, otras mujeres de la familia podían hacerlo. La madre de Gigi, por ejemplo, también le preparó baños a lo largo del embarazo, aunque no era una de las mujeres más viejas de esa familia extensa (entrevista, aldea Kwatá, 5 de noviembre de 2010).
Las prácticas de autoatención reconocidas por los munduruku por su eficacia en el embarazo y el parto son centrales en la reproducción biosocial de la colectividad, aunque las mujeres no quedan restringidas sólo a las prácticas consideradas tradicionales. En Dias-Scopel, Scopel y Langdon (2017) describimos la actuación del EMSI en el programa prenatal y la participación de las mujeres munduruku. Por ello, basta apuntar que las embarazadas, sus familiares, los curadores payés, las parteras y las mujeres mayores de la familia extensa no percibían a los miembros del EMSI como antagonistas sino como un recurso más, disponible en la aldea.

En este escenario de pluralismo médico, entre los actores sociales participantes en el proceso de atención del embarazo y el parto destaca la actuación de las mujeres mayores de la familia extensa, quienes ocupan un lugar primordial en las prácticas de autoatención.

\section{EN EL PARTO}

Los relatos de mujeres de diferentes edades, incluso de aquellas cuya experiencia de parto fue anterior a la presencia de los servicios biomédicos en la Tierra Indígena, permiten identificar una pluralidad de actores que auxilian en el parto domiciliario. Entre los munduruku, el nacimiento de un niño requiere la participación de varias personas, en especial, el cónyuge y una mujer mayor de la familia extensa, como la madre, la tía, la abuela o la suegra de la parturienta. Las mujeres mayores de la familia asumen la función de "recibir al niño" e influyen en la decisión de solicitar o no la ayuda de personas de fuera de la familia extensa si hay complicaciones. En esos casos, se recurre a las parteras o curadores payés, por ser personas experimentadas y con don para resolver trabajos de parto complicados. En fechas más recientes, en los relatos se menciona la participación de técnicos de enfermería y médicos. Todos 
desempeñan un papel de ayuda en el trabajo de parto domiciliario.

A continuación, analizamos un parto para mostrar la capacidad de los munduruku para articular las prácticas tradicionales con la actuación del equipo biomédico de salud y su apertura hacia los servicios biomédicos, pues esos procesos no suplantan la agencia y las prácticas indígenas.

Elis narró su parto tres días después del nacimiento de su sexto hijo, en noviembre de 2010, en el que participaron varios actores sociales: el cónyuge, la partera y el médico del EMSI (entrevista con Elis, aldea Kwatá, 15 de noviembre de 2010). El matrimonio de Elis es de tipo virilocal, es decir, la esposa pasa a vivir cerca de la familia del marido después del matrimonio, por lo tanto, su madre y sus hermanas viven en otra aldea. Bia, reconocida como partera de gran experiencia, es esposa del tío del marido de Elis. Las mujeres no se reconocían como parientes. El análisis de las genealogías reveló la relación de afinidad entre parturienta y partera, que ocupaban el mismo segmento residencial, organizado a partir de la consanguinidad de los maridos, en este caso específico. Con 69 años de edad, Bia era una de las mujeres más viejas del segmento residencial y vivía a unos $50 \mathrm{~m}$ de Elis.

En el relato de Elis, su cónyuge la tomó por la espalda para proporcionarle apoyo para sentarse en un pequeño banco utilizado para parir. Ésta es la posición de parto considerada tradicional por los munduruku. Sin embargo, identificamos otras posiciones de parto en las narraciones: de rodillas con las manos apoyadas en la hamaca; parcial o totalmente sentada en el suelo, con alguien que sostiene por la espalda con los brazos alrededor de la mujer; sentada en un taburete. Bia preparó el lugar del parto y cubrió el banco con telas. Desde la perspectiva de Elis, Bia recibió al bebé. Narró que estaba feliz por la presencia de John, el médico, pues el bebé nació con el "cordón enrollado en el cuello". El médico examinó al niño, le hizo asepsia a Elis y se fue. Entonces Bia haló la madre del cuerpo y ayudó a Elis en las tareas domésticas durante tres días. La actuación de una partera no se restringe al momento del parto y puede extenderse más allá de los procedimientos técnicos específicos, para cuidar de la casa y del recién nacido durante el posparto, si es necesario (entrevista con Elis, aldea Kwatá, 15 de noviembre de 2010).

El caso de Elis muestra una situación poco frecuente entre los munduruku, en la que participan una partera experimentada y un médico. La actividad de John era limitada, sus estadías de trabajo eran de 20 días al mes, por la extensión geográfica y la distribución de las aldeas en la TIKL, que no cuentan con servicio de telefonía o radiofonía. La actuación conjunta del médico y la partera era positiva, según John, quien reconocía el "saber empírico" de ellas. Éste era su primer empleo como médico. A pesar de que tenía 39 años de edad, era recién graduado en la Universidad Federal del Amazonas. Afirmaba haber crecido en una zona rural muy pobre y haber trabajado para pagarse los estudios. También decía que cumplía una "promesa de trabajar dos años en la salud indígena”, pues se había sensibilizado respecto a la "necesidad" de los indígenas durante su permanencia en la universidad (entrevista con John, aldea Kwatá, 31 de enero de 2010).

Bia dijo mantener una relación estrecha con los profesionales de la salud del EMSI para la atención de partos domiciliarios en la aldea Kwatá. Narró su participación en otro parto, a petición de la enfermera Suzy. Aunque Bia afirma que ya no asiste más partos por la edad y porque sufre de cataratas, cuenta que se sintió alentada por la enfermera, quien aseaba a la parturienta mientras ella tomaba al niño y le cortaba el cordón umbilical (entrevista con Bia, aldea Kwatá, 22 de marzo de 2011). Suzy tenía más de diez años trabajando en la salud indígena, primero como técnica de enfermería entre los mura, y después de graduarse, entre los munduruku, como jefa del equipo. Afirmó que "no tenía experiencia en partos" y 
solicitaba la presencia de Bia en partos domiciliarios (entrevista con Suzy, aldea Kwatá, 22 de abril de 2011). Esta apertura del médico y la enfermera hacia el trabajo de la partera refleja la posición de respeto de ambos, al menos en cuanto a la atención de partos. Los dos ocupaban posiciones de liderazgo y ejercían una influencia importante sobre los demás técnicos del EMSI, aunque éstos tendían a competir con los saberes indígenas y negar su validez.

La narración de un parto asistido por Araci, quien no se identifica como partera, comenzó después de que le preguntamos quién ayudó a Naná en el parto:

Yo, Iara, la tía de la parturienta y José, el muchacho, el técnico de enfermería del polo base.
Entonces, cuando ella estaba sufriendo con los dolores, ¿no?, nosotros ayudándola. Entonces, vimos que no iba a bajar el niño, así, de cuclillas como ella lo quería tener, ¿no? Ahí, incluso, creo que ella estaba nerviosa también, la atacó un nerviosismo en las piernas de ella, que ella se quedó así [mueve las piernas como si estuviera temblando]. No sé si es porque ella no se había alimentado bien antes, ¿no? Ahí, nosotros hicimos la cama y la colocamos. Entonces ella se acostó. Entonces vino pronto. El niño salió rápido, no costó. Ahora, como yo estoy diciendo, lo que sí dio trabajo fue la placenta, que ella tuvo siete horas [el bebé], se fue para tener nueve horas [la placenta]. Entonces, la arreglamos, arreglamos, arreglamos [en referencia a masajes abdominales]. Entonces, hay una planta aquí que

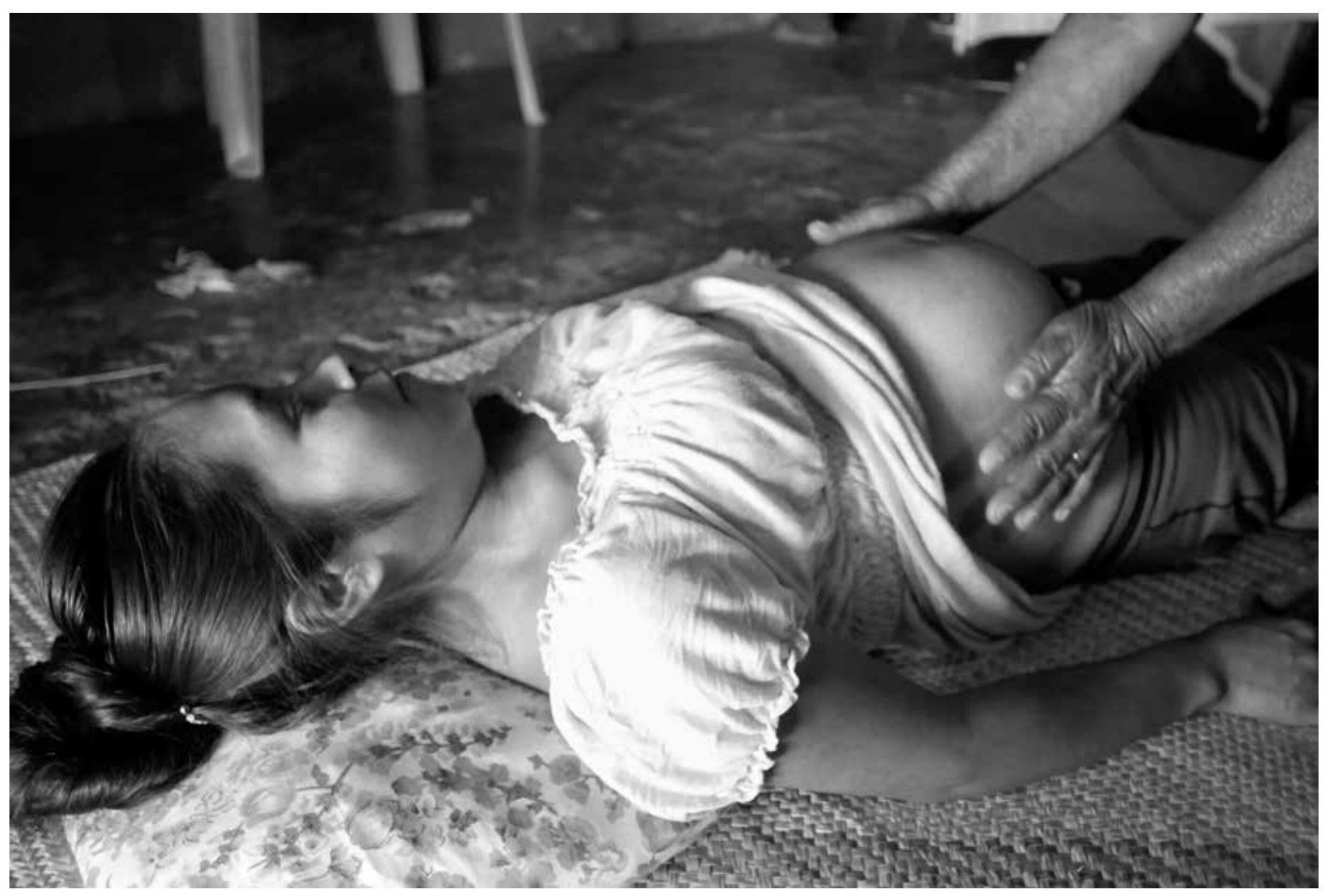

Consuelo Morales Pagaza • Francisca Morales, partera, ausculta el vientre de la mujer para cerciorarse de que el bebé viene en buena posición. Chilapa, Guerrero, 2017. 
usted conoce, cubiu, ¿no? Una planta así que da una fruta con la que hacemos dulce. ¡Allí en la casa hay! Con una hoja ancha. Ahí, esa hoja, agarramos esa hoja, la pilamos, sacamos así dos dedos de jugo, del jugo de ella, le ponemos una pizquita de sal y amoniaco y le da a beber a la persona. Entonces, yo cogí y le di, ¿no? Le di dos veces a ella. Entonces miré el reloj y yo decía: ¡ay, ay, ay, señor, nada que baja! Entonces, recordé la oración de santa Margarita. Ahí me quedé, detrás de la espalda de ella. Ahí fui hablando: "voy a decir una oración y quiero que tú repitas conmigo", le dije a ella. Entonces yo decía: "santa Margarita [...]”, de ahí, yo iba diciendo, de ahí, ella iba diciendo. Yo rezaba tres veces detrás de la espalda de ella. Fue terminando la oración y aquello fue bajando. Ella la tuvo rapidito [la placenta] (entrevista con Araci, aldea Kwatá, 13 de mayo de 2011).

Después de que el bebé nació, limpiaron a la parturienta y el lugar del parto con la colaboración de José, el cónyuge, quien enterró la placenta y las telas sucias de sangre. Esto es importante para no atraer los espíritus-animales de los delfines malignos. Cuando José llegó, el bebé ya estaba naciendo. Antes de irse, el técnico de enfermería solicitó que prepararan un vaso de leche para la parturienta (entrevista con $\mathrm{Na}-$ ná, aldea Kwatá, 14 de mayo de 2011). Dar masajes al cuerpo materno después del parto se considera esencial para la salud de la mujer. Este parto terminó cuando la mujer mayor, que comandaba la escena, haló la madre del cuerpo de la parturienta.

Cabe señalar que la participación de las parteras y las mujeres más viejas de las familias extensas no se distingue por sus prácticas, sino por el reconocimiento que se les otorga, tanto por parte de la comunidad como de algunos profesionales de la salud. Recordemos que quienes son reputadas como parteras también son las mujeres más viejas de su segmento residencial. Así, mientras las mujeres mayores de la familia extensa actúan en el interior de la familia, las parteras pueden circular entre varias familias extensas. A partir de las narrativas, se observa que las parteras son llamadas a atender partos complicados, demorados o con el niño atravesado. Cuando se prevé que habrá complicaciones, la mujer mayor de la familia extensa suele llamar a otra mujer para que le ayude. En la aldea Kwatá, las mujeres identificadas como parteras fueron reconocidas como actores sociales que colaboraban en partos difíciles. Por ejemplo, Rita narra que tuvo uno de sus diez hijos con el auxilio de su cuñada Araci, una de las mujeres más viejas de la familia extensa, y la partera Bia. Rita estaba embarazada de gemelos y uno de ellos estaba sentado. Su cuñada no estaba teniendo éxito en ese parto difícil y pidió ayuda a Bia, quien vivía en otro segmento residencial, a unos cientos de metros de la casa de Rita. Ambas decidieron llevar a Rita al hospital de la ciudad. Bia acompañó a la embarazada en el viaje en lancha, aun sin ser parientes. A la mitad del camino, según la partera, Rita se quejó de dolor y agotamiento. Relató que "no aguantaba más” y que Bia pidió al conductor de la lancha que atracara a la orilla del río y atendió el parto "allí mismo", en la lancha. El primer bebé, según la madre, "nació sentado", "de culo". Después del parto, siguieron el viaje a la ciudad (entrevista con Rita, aldea Kwatá, 6 de septiembre de 2017).

Maiara, de 64 años de edad, quien se identifica y es identificada como partera, también narró casos de partos difíciles. Citó el parto de su cuñada, cuyo hijo "nació de culo", a pesar de todo el esfuerzo para enderezar al bebé aún en el vientre, por medio de masajes con agua tibia y aceite de copaíba. El hijo de una sobrina atendida por Maiara murió poco después del parto. La partera cuenta que el bebé tenía una "cabeza muy grande" y sobre ella, en lugar de la mollera, había algo que "parecía una boca”, que se abría y cerraba. En ese caso, la muerte del recién nacido se justificó por ser un hijo de animal — delfín— (entrevista con Maiara, aldea Kwatá, 
7 de septiembre de 2017). En el campo oímos otras narraciones sobre hijos de animal que murieron poco después del parto. En general, estos bebés se describen con deformaciones, la mitad del cuerpo con apariencia humana y la otra mitad animal. Maiara narró el parto de una de sus hijas, cuyo bebé nació muerto, "de culo". Además de atender partos y agarrar la barriga, Maiara también "arregla huesos torcidos", "reza contra espina", "bendice mal de ojo" y "hace remedio" para niño, mujer menstruada y mujer en dieta de posparto (entrevista con Maiara, aldea Kwatá, 7 de septiembre de 2017).

En el caso munduruku, las parteras son personas reconocidas como especialistas en la atención tradicional de la gestación y el parto, en particular en casos complicados, y como poseedoras de un conocimiento amplio sobre el cuerpo femenino. Ellas actúan más allá del segmento residencial propio y la familia extensa. Sin embargo, su intervención no es obligatoria. Al observar el proceso de nacimiento desde la gestación, en la atención a la gestante aparece con frecuencia una de las mujeres mayores de la familia extensa. Esta mujer puede no ser reconocida como partera fuera de su propia familia.

Los datos apuntan a que, en general, en la sociedad munduruku los saberes centrales para la atención del embarazo, el parto y la salud de la mujer se comparten y no están restringidos a la actuación de las mujeres reconocidas como parteras.

\section{¿Quiénes son las parteras munduruku?}

En el trabajo de campo, preguntamos a los munduruku quiénes eran las parteras en la aldea Kwatá. Conseguimos que indicaran cinco mujeres. Todas tenían más de 50 años de edad, cada una pertenecía a una familia extensa y vivían en distintos segmentos residenciales. Esa cantidad de parteras no equivale a la multiplicidad de mujeres que participa en la atención del embarazo y el parto. Por ejemplo,
Araci, que atendió embarazadas - incluso a Raquel, la investigadora- y partos en el transcurso del trabajo de campo, no fue identificada ni se identificó como partera.

La cuestión que se plantea es la siguiente: ¿por qué algunas mujeres que participaban en la atención de embarazos y partos no fueron identificadas como parteras por los habitantes de la aldea? Las cinco mujeres parteras, según los residentes de la aldea y AIS a quienes entrevistamos (aldea Kwatá, 1 de febrero de 2010), han participado en cursos de capacitación ofrecidos por la Fundación $\mathrm{Na}$ cional de Salud (Funasa). Esto nos llevó a pensar que la participación en cursos de capacitación sería un criterio importante para la identificación de las parteras. Sin embargo, cuando entrevistamos a esas mujeres, constatamos que sólo dos de ellas tomaron un curso de capacitación biomédica. No recordaban con exactitud cuándo participaron, pero sí los contenidos generales, como la higiene en el parto y el cuidado del material para cortar el cordón umbilical y asear a la parturienta. También hicieron una analogía entre las instrucciones de asepsia biomédica y las prácticas indígenas de cuidado en relación con las telas sucias de sangre del parto, y rememoraron la importancia de no lavarlas en la orilla del río como una práctica de higiene relativa al parto que evita la aproximación de los delfines malignos. Según Jaci, de 75 años de edad, una de las parteras que tomó el curso recibió un "libro de la partera" (entrevista con Jaci, aldea Caioé, 8 de septiembre de 2017).

Verificamos si el señalamiento de las mujeres como parteras se basaba en que habían participado en el curso de capacitación. Con base en los resultados que obtuvimos, proponemos que, entre los munduruku, estos cursos se mencionan como una señal diacrítica para la identificación de las parteras, pero no legitiman su actuación como tales. Para los munduruku, la legitimidad se sostiene por la noción de don. Todas las mujeres indígenas entrevistadas, que en algún momento atendieron la 
gestación y el parto de otras mujeres, afirmaron que este conocimiento es un don. Los curadores payés, las parteras y mujeres mayores son ejemplos de indígenas que pueden tener el don para agarrar la barriga y "partear", habilidades consideradas inherentes a la persona desde el nacimiento, que se manifiestan en la práctica y la experiencia en casos concretos. El don es una forma de saber corporeizado, asociado a ciertas habilidades y experiencias que se desarrollan a lo largo de la vida, en los pasajes etarios y la iniciación chamánica. Scopel (2013) hace una reflexión más detallada sobre la categoría don entre los munduruku. Por el momento, es suficiente señalar que se trata de una forma de valorar un saber nato y de afirmar que ese tipo de conocimiento no proviene de libros, lo que marca una diferencia respecto al modo occidental de transmisión de conocimiento por la escritura. El don, en ese sentido, sintetiza el modo en que se manifiesta en la localidad un sistema social de adquisición de habilidades y construcción de legitimidades.

La identificación inicial de sólo cinco parteras también puede relacionarse con la construcción de la legitimidad de los actores sociales, marcada por el entrenamiento biomédico y evocada en un diálogo con investigadores y profesionales de la salud en un contexto de pluralidad médica. Para los munduruku, la capacitación biomédica es un complemento y no coincide con el don para ser partera. Las mujeres que recibieron capacitación biomédica fueron nombradas justo por el reconocimiento y notoriedad de su experiencia en la atención de partos. De hecho, la legitimidad social fue el criterio utilizado para seleccionar a las personas que recibirían la capacitación.

Aunque sólo dos de las mujeres identificadas como parteras hubieran tomado un curso de capacitación, las cinco señaladas tenían legitimidad social para actuar como parteras por ser poseedoras del don para agarrar la barriga, halar la madre del cuerpo, atender partos y recetar baños, rezos e

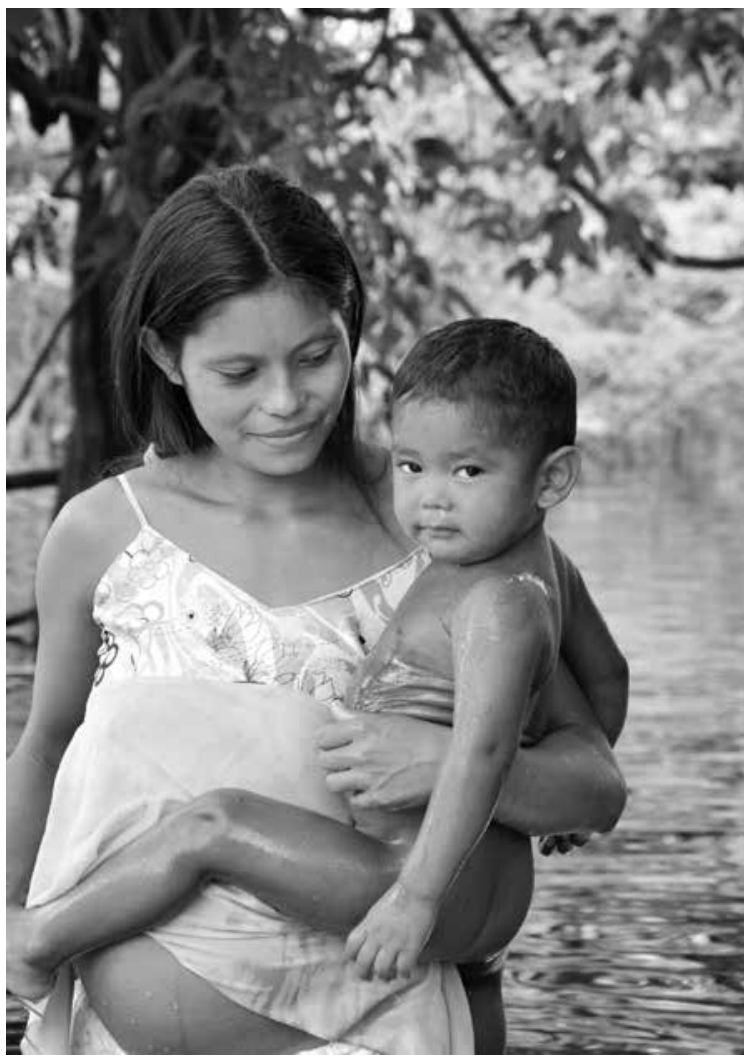

DANIEL Scopel • Gestante munduruku en la orilla del río Canumã, después de bañar a su hijo menor. Aldeia Kwatá, abril de 2011.

infusiones. Por otra parte, estas cinco parteras también dominaban otras prácticas, con frecuencia referidas a los curadores payés, como arreglar huesos torcidos, coser y bendecir. El dominio de ciertas prácticas no se restringe a la categoría partera, pues recibir al niño — prestar asistencia al parto- es sólo una de las prácticas de estas mujeres. De la misma manera, recibir al niño no es exclusivo de las parteras, pues las mujeres mayores de las familias extensas también lo hacen y gozan de legitimidad social entre los familiares que reconocen su don, aunque no se identificasen como parteras.

La autoidentificación como partera implica “asumir responsabilidades” y “compromiso”, según explica Bia (entrevista, aldea Kwatá, 6 de septiembre 
de 2017), porque los miembros de la familia elemental temen, pues la muerte es uno de los riesgos presentes en un parto, como explicó la partera Maiara (entrevista, aldea Kwatá, 7 de septiembre de 2017). Araci, que atiende embarazos y partos entre los miembros de su familia extensa, explicó que "no dice que es partera" porque asumir este papel es encarar la disponibilidad para atender los llamados de muchas mujeres y aceptar responsabilidades (entrevista con Araci, aldea Kwatá, 8 de septiembre de 2017). Todas las parteras reconocieron que no negarían ayuda cuando fueran llamadas, pues la muerte de la madre y el bebé puede entenderse como culpa de ellas, incluso cuando no hay nada que hacer. El parto no sólo es sinónimo de vida, sino también de muerte, y eso trae consecuencias para la autoidentificación de las parteras.

De las cinco parteras identificadas, sólo Jaci afirmó que atendía partos todavía. Jaci había atendido el parto de una nuera hacía menos de 20 días, en septiembre de 2017. Ella y otras mujeres estaban sentadas bajo la sombra de un árbol, incluida la nuera, en dieta de posparto y con su bebé, cuando Jaci narró algunos casos. Cuando la investigadora comentó “ciertamente, usted ya ha atendido más de 100 partos", Jaci señaló que en los partos de esas "nueras todo es yo", y añadió: "mis hijos ya tienen mujeres e hijas mujeres que yo atendí" (entrevista con Jaci, aldea Caioé, 8 de septiembre de 2017). Aunque es una de las parteras que tomó un curso de capacitación biomédica y en ocasiones atiende a otras familias extensas, se observa que el centro de su práctica está en el interior de su propia familia extensa, en la que es una de las mujeres más viejas. Así, podemos sugerir que la intervención de las parteras en otras familias extensas no es una regla, sino un caso de excepción marcado por dificultades.

Hay otros casos en los que se acude a las parteras; por ejemplo, cuando los miembros del EMSI tienen que atender un parto en la aldea. Aunque Bia afirmó que ya no atendía, en 2011 apoyó al EMSI en dos partos. Uno fue en la casa de la parturienta y el otro en el polo base (entrevista con Bia, aldea Kwatá, 2 de septiembre de 2017). Maiara también relató que había atendido casos en el polo base, a petición de las técnicas de enfermería y la enfermera (entrevista con Maiara, aldea Kwatá, 7 de septiembre de 2017). Sin embargo, todas las parteras afirman que "después del polo base", es decir, del establecimiento y la actividad del EMSI en la aldea Kwatá, muchas mujeres optan por parir en el hospital público de la ciudad.

Hasta qué punto el EMSI intenta instrumentalizar la participación de las parteras para hacer efectiva su posición de poder en el momento del parto domiciliario en la aldea es una cuestión que debe profundizarse en investigaciones futuras. Por ahora, señalamos que incluso en los contextos de pluralidad médica se verifica la creciente expansión de la medicalización del parto entre los pueblos indígenas brasileños, focos de una lógica instrumental orientada hacia la universalización de la atención primaria.

\section{Consideraciones finales}

Las prácticas de autoatención reconocidas por su eficacia en el embarazo y el parto entre los munduruku tienen importancia central en la reproducción biosocial colectiva. Se trata de un saber difundido y no restringido a la destreza de parteras y curadores payés.

Durante el embarazo, los munduruku manipulan la barriga de la embarazada con masajes - agarrar la barriga-, para prevenir problemas e investigar el sexo, posición y tiempo de gestación del feto. Además de la cuestión anatómica y fisiológica, tratan de descifrar asuntos relativos a la cosmología, como la posibilidad de gestación generada por el contacto inadecuado con espíritus-animales maléficos. También utilizan baños con plantas para facilitar la expulsión del bebé. Estas cuestiones no 
implican oposición a la atención biomédica, pues las mujeres munduruku recurren a estos servicios ofrecidos por el Estado para empezar el control prenatal (Dias-Scopel, Scopel y Langdon, 2017).

En el parto domiciliario participan varias personas, en especial el cónyuge y una mujer mayor de la familia extensa, que puede ser reconocida como partera o no. Sin embargo, en la actualidad, el equipo de salud biomédica recomienda que casi la mitad de los partos se atienda en el hospital, y los partos domiciliarios pueden contar o no con la participación de técnicos de enfermería o enfermeros, lo que depende de su presencia en la aldea. En partos difíciles, se recurre a las parteras más experimentadas, quienes ayudan a evaluar la posibilidad de conducir a la parturienta al hospital más cercano. La muerte siempre es un riesgo que las parteras asumen.
Entre las mujeres que tienen el don para atender partos, sólo cinco fueron identificadas como parteras por los moradores de la aldea. Ellas tienen la legitimidad para circular entre varias familias extensas debido al reconocimiento social que poseen y su experiencia en partos difíciles, en situaciones que involucran la muerte y relaciones con seres invisibles y espíritus-animales. Ellas poseen el don, una forma de saber corporeizado, que asocia habilidades y experiencias desarrolladas en los pasajes etarios y la iniciación chamánica, que no se confunde con el saber aprendido en libros y cursos. Algunas de estas mujeres han participado en capacitaciones biomédicas, que se consideran complementos del don.

Desde la primera Conferencia de Salud Indígena en Brasil, en 1986 (citado en Fundação Nacional de Saúde, 2002), se enfatizó la necesidad de

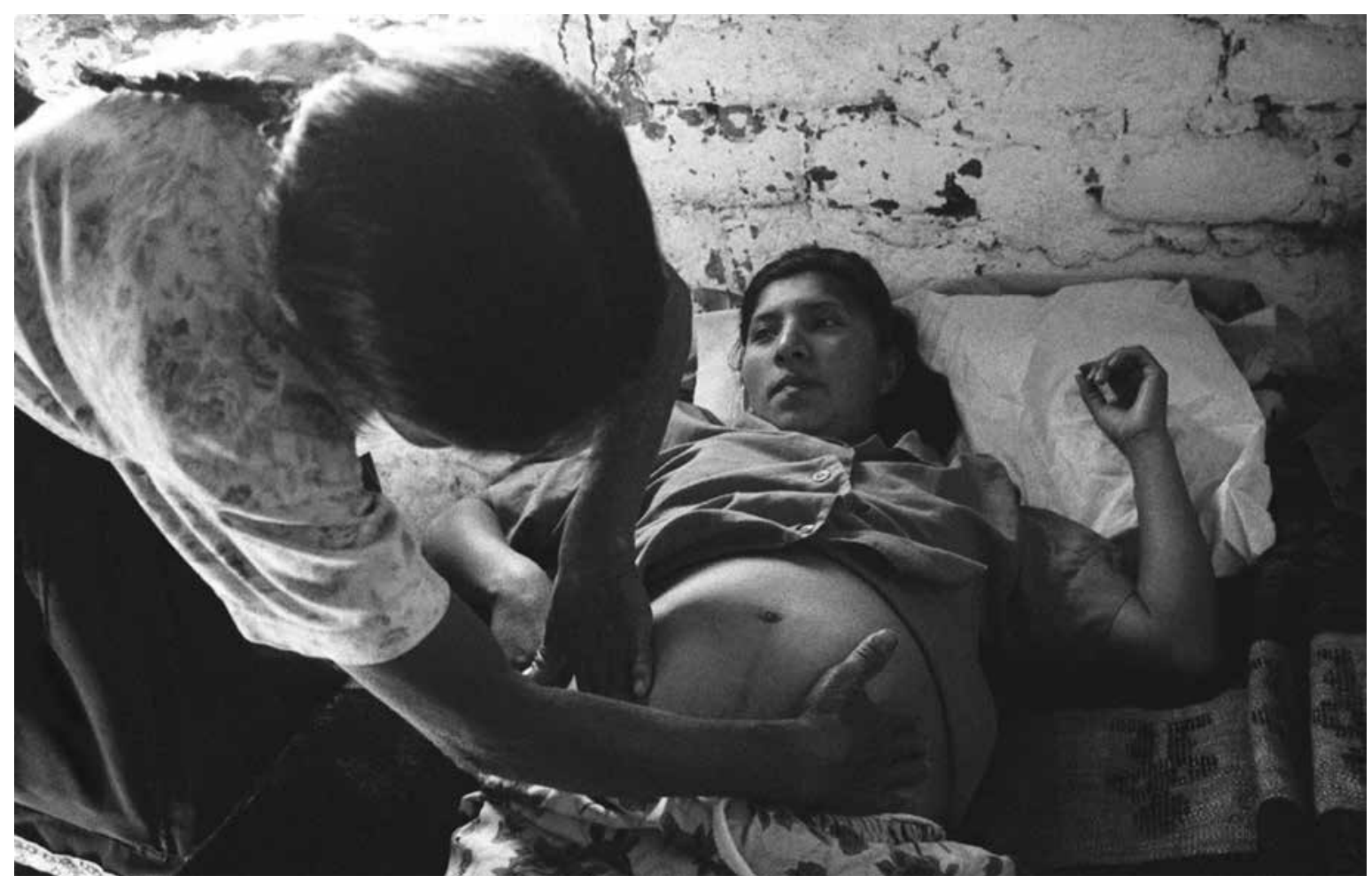

RICARDo RAmirez ARRIola P Por su labor, experiencia y sabiduría, las comadronas son muy valoradas y tienen poder e influencia sobre las demás mujeres de la comunidad. San Pedro Pinula, Jalapa, Guatemala, 2000. 
promover la atención diferenciada para mejorar la calidad y eficacia de los servicios de salud prestados a las poblaciones indígenas, con respeto hacia la diversidad de saberes y practicantes involucrados. Sin embargo, al considerar los riesgos del parto domiciliario, en ocasiones los profesionales de la salud incentivan el parto hospitalario y desalientan el trabajo de las parteras. Esto contradice los ideales del programa Trabajando con Parteras Tradicionales, promovido por el MS, y refuerza la expansión de la medicalización del parto en contextos de pluralidad médica.

Entre los munduruku, las parteras y mujeres mayores de la familia extensa intervienen desde que comienza el embarazo hasta el posparto; sus actividades no se restringen al momento del parto y abarcan cuidados relacionados con la salud de la mujer y el niño desde una perspectiva chamánica del cosmos y el cuerpo según los saberes reconocidos como tradicionales. Las prácticas indígenas de autoatención y la atención prestada por las parteras a las mujeres munduruku coexisten con la presencia de profesionales biomédicos del servicio oficial de salud en las aldeas, lo que configura un escenario complejo que no se reduce a la simple oposición o antagonismo entre las formas de atención tradicional y biomédica. Como se ve en este artículo, la pluralidad puede también producir articulaciones entre actores sociales con potencial para evitar o al menos minimizar la tendencia a la hospitalización del parto en el contexto indígena. D

\section{Bibliografía}

Dias-Scopel, Raquel Paiva, 2015, A cosmopolitica da gestação, do parto e do pós-parto: práticas de autoatenção e o processo de medicalização entre os indios munduruku, Associação Brasileira de Antropologia/Gesellschaft für Internationale Zusammenarbeit/Paralelo 15, Brasilia.

Dias-Scopel, Raquel Paiva, Daniel Scopel y Esther Jean Langdon, 2017, "Gestação, parto e pós-parto entre os munduruku do Amazonas: confrontos e articulações entre o modelo médico hegemônico e práticas indígenas de autoatenção", en I/ha, Revista de Antropologia, vol. 19, núm. 1, pp. 183-216.

Ferreira, Luciane Ouriques, 2013, Medicinas indigenas e as politicas da tradição: entre discursos oficiais e vozes indigenas, Fundação Oswaldo Cruz, Río de Janeiro.

Fundação Nacional do Índio (Funai) y Ministério da Justiça, 2002, “Mapa de demarcação Terra Indígena Kwatá-Laranjal”. Disponible en línea: <http://mapas2.funai.gov.br/portal_mapas/pdf/terra_indigena.pdf>.

Fundação Nacional de Saúde, 2002, Política nacional de atenção à saúde dos povos indígenas, Ministério da Saúde, Brasilia.

Garnelo, Luiza, 2003, Poder, hierarquia e reciprocidade: saúde e harmonia entre os Baniwa do Alto Rio Negro, Fundação Oswaldo Cruz, Río de Janeiro.

Ghiggi Junior, Ari, 2015, Uma abordagem relacional da atenção à saúde a partir da terra indígena Xapecó, tesis de doctorado en antropología social, Universidade Federal de Santa Catarina, Florianópolis.

Langdon, Esther Jean, 2007, “Problematizando os projetos de medicina tradicional indígena”, en Medicina tradicional indígena em contextos. Anais da I reunião de monitoramento, Luciane Ouriques Ferreira y Patricia Silva Osório (orgs.), Ministério da Saúd-Fundação Nacional de Saúde-Projeto Vigisus II, Brasilia, pp. 110-119.

Menéndez, Eduardo, 1987, “Medicina tradicional o sistemas práctico-ideológicos de los conjuntos sociales, como primer nivel de atención”, en Centro Interamericano de Estudios de Seguridad Social (CIESS), El futuro de la medicina tradicional en la atención a la salud de los paises latinoamericanos, Centro Interamericano de Estudios de Seguridad Social, México, pp. 37-62.

—_ 2009, Sujeitos, saberes e estruturas: uma introdução ao enfoque relacional no estudo da saúde coletiva, Hucitec, São Paulo.

Ministério da Saúde, 2010, Parto e nascimento domiciliar assistidos por parteiras tradicionais. O Programa Trabalhando com Parteiras Tradicionais e Experiências Exemplares, Ministério da Saúde-Secretaria de Atenção à Saúde, Brasilia. 
Scopel, Daniel, 2013, Uma etnografia sobre a pluralidade de modelos de atenção à saúde entre os índios munduruku na Terra Indigena KwatáLaranjal, Borba, Amazonas: práticas de automação, xamanismo e biomedicina, tesis de doctorado en antropología social, Universidade Federal de Santa Catarina, Florianópolis.

Scopel, Daniel, Raquel Paiva Dias-Scopel y Flávio Braune Wiik, 2012, "Cosmologia e intermedicalidade: o campo religioso e a autoatenção às enfermidades entre os índios munduruku do Amazonas, Brasil”, en Tempus: Actas de Saúde Coletiva, vol. 6, núm. 1, pp. 173-190.

Scopel, Daniel, Raquel Paiva Dias-Scopel y Esther Jean Langdon, 2015, "Intermedicalidade e protagonismo: atuação dos agentes indígenas de saúde munduruku da Terra Indígena Kwatá-Laranjal, Amazonas, Brasil”, en Cadernos de Saúde Pública, vol. 31, núm. 12, pp. $2559-2568$.

__ 2018, "A cosmografia munduruku em movimento: saúde, território e estratégias de sobrevivência na Amazônia Brasileira”, en Boletim do Museu Paraense Emílio Goeldi. Ciências Humanas, vol. 13, núm. 1, pp. 89-108.

Silva, Sandra Cavalcante, 2017, Atenção à gestação e ao parto em uma comunidade rural amazônica: reflexões sobre o papel da parteira, tesis de maestría, Instituto Leônidas e Maria Deane/Fundação Oswaldo Cruz, Manaos.

Wakanã, Graciliana Selestino y Laura Pérez Gil, 2007, "Caracterização dos sistemas de parto tradicionais entre os povos indígenas de Alagoas e Pernambuco: resultados da primeira etapa”, en Luciane Ouriques Ferreira y Patricia Silva Osório (orgs.), Medicina Indígena em Contextos. Anais da I Reunião de Monitoramento, Ministério da Saúd-Fundação Nacional de Saúde-Projeto Vigisus II, Brasilia, pp. $37-47$.

\section{Entrevistas}

Alice, aldea Laranjal, 16 de febrero de 2010.

Araci, aldea Kwatá, 13 de mayo de 2011 y 8 de septiembre de 2017.

Bia, aldea Kwatá, 22 de marzo de 2011, 2 y 6 de septiembre de 2017.

Elis, aldea Kwatá, 15 y 17 de noviembre de 2010.

Enfermera y médico, aldea Kwatá, 31 de enero de 2010.

Jaci, aldea Caioé, 8 de septiembre de 2017.

John, aldea Kwatá, 31 de enero de 2010.

Madre de Gigi, aldea Kwatá, 5 de noviembre de 2010.

Maiara, aldea Kwatá, 7 de septiembre de 2017.

Naná, aldea Kwatá, 14 de mayo de 2011.

Residentes de la aldea y Als, aldea Kwatá, 1 de febrero de 2010.

Rita, aldea Kwatá, 6 de septiembre de 2017.

Suzy, aldea Kwatá, 22 de abril de 2011. 